University of Nebraska - Lincoln

DigitalCommons@University of Nebraska - Lincoln

John R. Hardy Papers

Research Papers in Physics and Astronomy

10-15-1974

\title{
Second-Order Raman Scattering in Alkali Fluoride Crystals
}

S. L. Cunningham

University of Nebraska - Lincoln

T. P. Sharma

University of Nebraska - Lincoln

Sitaram Jaswal

University of Nebraska, sjaswal1@unl.edu

M. Hass

University of Nebraska - Lincoln

John R. Hardy

University of Nebraska - Lincoln

Follow this and additional works at: https://digitalcommons.unl.edu/physicshardy

Part of the Physics Commons

Cunningham, S. L.; Sharma, T. P.; Jaswal, Sitaram; Hass, M.; and Hardy, John R., "Second-Order Raman Scattering in Alkali Fluoride Crystals" (1974). John R. Hardy Papers. 15.

https://digitalcommons.unl.edu/physicshardy/15

This Article is brought to you for free and open access by the Research Papers in Physics and Astronomy at DigitalCommons@University of Nebraska - Lincoln. It has been accepted for inclusion in John R. Hardy Papers by an authorized administrator of DigitalCommons@University of Nebraska - Lincoln. 


\title{
Second-order Raman scattering in alkali fluoride crystals*
}

\author{
S. L. Cunningham, ${ }^{\dagger}$ T. P. Sharma, ${ }^{\ddagger}$ S. S. Jaswal, M. Hass, ${ }^{\S}$ and J. R. Hardy \\ Behlen Laboratory of Physics, University of Nebraska, Lincoln, Nebraska 68508
}

(Received 19 February 1974)

\begin{abstract}
We present theoretical and experimental second-order Raman spectra for NaF, KF, RbF, and CsF. The theoretical calculation follows the formalism of Born and Bradburn. For the lattice-dynamic calculation we use the deformation-dipole model and present comparisons with measured dispersion curves. For the polarizability calculation, we retain all eight first-neighbor and all thirty second-neighbor polarizability coefficients. We find that, in addition to all of the first-neighbor coefficients, only three of the second-neighbor coefficients are needed to obtain good agreement between the calculated and observed spectra both as regards peak positions and over-all shape. Comparison with other types of calculations are made.
\end{abstract}

\section{INTRODUCTION}

The basic treatment for calculating the secondorder Raman scattering was worked out in 1947 by Born and Bradburn ${ }^{1}$ and applied to $\mathrm{NaCl}$. Shortly thereafter, $\mathrm{Smith}^{2}$ applied the theory to diamond. Because the theory requires rather complete knowledge of the phonons, further work was delayed until the development of the computer. Then Cowley ${ }^{3}$ in 1964 and Hardy and Karo in a series of papers in collaboration with others, ${ }^{4,5}$ from $1965-1969$ performed calculations for various alkali halides. During this period, the calculations were compared with spectra that were recorded by photographic plate. This had the disadvantage that the intensity scale was highly nonlinear and thus comparisons between experiment and calculation could only be made on a qualitative basis.

With the introduction of the laser as a tool for studying Raman spectra and with the use of photoncounting techniques for obtaining linear intensity measurements, it became possible after 1970 to attempt to get quantitative agreement between calculations and experiment. Thus, we find recent work by Cunningham, Hardy, and Hass, ${ }^{6}$ Bruce and Cowley, ${ }^{7}$ Pasternak, Cohen, and Gilat, ${ }^{8}$ Krauzman, ${ }^{9}$ Jaswal, Wolfram, and Sharma, ${ }^{10}$ and Sharma, Kirby, and Jaswal ${ }^{11}$ in which a concentrated effort is made to obtain quantitative agreement between calculation and experiment. This paper reports the comparison between theory and experiment for four of the alkali fluorides, namely, NaF, KF, RbF, and CsF.

In Sec. II we present the results of Born and Bradburn ${ }^{1}$ and show how the theoretical calculation naturally splits into two parts: One is the determination of the phonon frequencies and eigenvectors and the other is the determination of the effect of each pair of phonons on the polarizability of the lattice. In Sec. III we briefly describe the lattice dynamic calculation. In Sec. IV we discuss the polarizability phase of the problem. It is this part of the calculation that distinguishes the various types of calculations in Refs. 6-10, and it is this part of the calculation upon which future work should concentrate. In Sec. $V$ we discuss the relation between the calculated spectra and the experimentally measured spectra, and in Sec. VI we present the results.

\section{SCATTERING INTENSITY}

The intensity of light inelastically scattered from a system of phonons into a solid angle $d \Omega$ is given by ${ }^{1}$

$$
I\left(\omega_{f}\right) d \Omega=\frac{\omega_{i}^{4}}{2 \pi c^{3}} \sum_{p=1}^{2} \sum_{\substack{\alpha \beta \\ \gamma \delta}} n_{\alpha}^{p} n_{\beta}^{p} i_{\alpha \beta \gamma \delta}(\omega) E_{\gamma}^{-} E_{\delta}^{+} d \Omega,
$$

where $\omega=\omega_{i}-\omega_{f}$, with $\omega_{i}$ and $\omega_{f}$ being the frequencies of the incident and scattered photons, respectively, and $c$ is the speed of light in vacuum. In this expression, $\vec{n}^{1}$ and $\vec{n}^{2}$ are two mutually perpendicular unit vectors that are also perpendicular to the direction of propagation of the scattered light; they are used to specify the polarization of the scattered radiation. The terms $E_{\gamma}^{-}$and $E_{B}^{+}$are components of the incident electric field. The greek subscripts $\alpha, \beta, \gamma$, and $\delta$ range over the three Cartesian components $x, y$, and $z$.

For second-order Raman scattering, the term $i_{\alpha \beta \gamma \delta}(\omega)$ can be written

$$
\begin{aligned}
i_{\alpha \beta \gamma \delta}(\omega)= & \frac{\hbar^{2}\left(\omega_{i}-\omega\right)^{4}}{8 \omega_{i}^{4}} \sum_{\vec{d} j j^{\circ}} \frac{T(\omega(\overrightarrow{\mathrm{q}} j)) T\left(\omega\left(\overrightarrow{\mathrm{q}} j^{\prime}\right)\right)}{\omega(\overrightarrow{\mathrm{q}} j) \omega\left(\overrightarrow{\mathrm{q}} j^{\prime}\right)} \\
& \times P_{\alpha \beta}\left(\overrightarrow{\mathrm{q}} \mid j j^{\prime}\right) P_{\gamma \delta}\left(\overrightarrow{\mathrm{q}} \mid j j^{\prime}\right) \delta\left(\omega \pm \omega(\overrightarrow{\mathrm{q}} j) \pm \omega\left(\overrightarrow{\mathrm{q}} j^{\prime}\right)\right),
\end{aligned}
$$

where $\omega(\vec{q} j)$ is the frequency of the phonon having wave vector $\vec{q}$ and belonging to the phonon branch $j$. Since $\omega$ is positive for the Stokes-shifted component, the + and - signs in the $\delta$ function correspond to the destruction and creation of a phonon, respectively. The prefactor $\left(\omega_{i}-\omega\right)^{4} / \omega_{i}^{4}$ is due to the fact that $\omega_{i}^{4}$ rather than $\omega_{f}^{4}$ was used as the prefactor in Eq. (1). Usually, this prefactor is as- 
sumed to be 1 , but we retain it in this work because $\omega / \omega_{i} \sim 0.06$ for the alkali fluorides and thus this term represents a correction which may change by as much as $20 \%$ over the range of frequency in the spectrum.

The temperature-dependent terms $T(\omega(\vec{q} j))$ result from the thermal averaging process and are given by

$$
T(\omega(\overrightarrow{\mathrm{q}} j))=\left\{\begin{array}{l}
n(\omega(\overrightarrow{\mathrm{q}} j))+1, \\
n(\omega(\overrightarrow{\mathrm{q}} j)),
\end{array}\right.
$$

depending upon whether the $(\overrightarrow{\mathrm{q}} j)$ phonon is created (upper) or destroyed (lower) in the scattering process; here

$$
n(\omega)=\left[e^{\hbar \omega / k_{B} T}-1\right]^{-1}
$$

is the usual Bose factor.

The term $P_{\alpha \beta}\left(\vec{q} \mid j j^{\prime}\right)$ is called the phonon polarizability. It represents the change in the $\alpha \beta$ component of the crystal polarizability tensor (which relates the polarization field to the external electric field) due to the presence of two phonons; one which has wave vector $\vec{q}$ and belongs to branch $j$ and the other which has wave vector $-\vec{q}$ and belongs to branch $j^{\prime}$. As we will see in Sec. IV, this phonon polarizability depends upon the phonon eigenvector.

The problem of calculating the second-order Raman spectra using this approach, then, reduces to two basic parts. The first part is the calculation of the set of phonon frequencies $\omega(\vec{q} j)$ and the associated eigenvectors $\sigma_{\alpha}(\kappa \mid \vec{q} j)$ appropriate for the crystal of interest for all values of the wave vector within the first Brillouin zone. The second part is the determination of the phonon-dependent polarizability coefficients $P_{\alpha \beta}\left(\vec{q} \mid j j^{\prime}\right)$. These parts are the subject of Secs. III and IV, respectively.

\section{LATTICE DYNAMIC CALCULATION}

The phonon frequencies and eigenvectors were determined using a form of the deformation-dipole model of Hardy. ${ }^{12}$ The model employed contains the following parameters: (i) $A, B$ : the firstneighbor, central, short-range force constants; (ii) $A^{+}, B^{+}, A^{-}, B^{-}$: the central short-range force constants for ++ and -- second neighbors, respectively; (ii) $C^{+}, C^{-}$: the angle-bending force constants; (iv) $\gamma_{ \pm}, \gamma_{ \pm}^{\prime}=A \gamma_{ \pm} / B$; the deformation-dipole parameters of $\mathrm{Hardy}^{12}$ which represent the dipole moment and its first derivative, respectively, as sociated with the deformation of the electron cloud around the positive and negative ions, and (v) $\alpha^{+}$, $\alpha^{-}$; the ionic polarizabilities of the positive and negative ions, respectively.

These model parameters are related to experimental quantities in the following way ${ }^{13}$ :

$$
\begin{aligned}
& c_{11}=\left(e^{2} / 2 r_{0}^{4}\right)\left[\frac{1}{2}\left(A+A^{+}+A^{-}+B^{+}+B^{-}\right)-2.5560\right], \\
& c_{12}=\left(e^{2} / 2 r_{0}^{4}\right)\left[\frac{1}{4}\left(A^{+}+A^{-}-B^{+}-B^{-}\right)\right. \\
& \left.+\frac{1}{3} \alpha_{m}+0.1131\right] \\
& c_{44}=c_{12}+\left(e^{2} / 2 r_{0}^{A}\right)\left(C^{+}+C^{-}\right) \text {, } \\
& B=-\frac{2}{3} \alpha_{m}-2\left(B^{+}+B^{-}\right) \text {, } \\
& \mu \omega_{\text {TO }}^{2}=\left[A+2 B+4\left(C^{+}+C^{-}\right)\right]\left(e^{2} / v_{a}\right) \\
& -\frac{4 \pi}{3 v_{a}}(e s)^{2}\left[1-\frac{4}{3} \pi\left(\frac{\alpha^{+}+\alpha^{-}}{v_{a}}\right)\right]^{-1}, \\
& \gamma_{ \pm}=\mp e B(1-s) /(2 A+4 B) \text {, }
\end{aligned}
$$

where

$$
s=\frac{3 \omega_{\mathrm{T}}\left(\mu v_{a}\right)^{1 / 2}}{e\left(\epsilon_{\infty}+2\right)}\left(\frac{\epsilon_{0}-\epsilon_{\infty}}{4 \pi}\right)^{1 / 2} .
$$

Here, $\left\{c_{\alpha \beta}\right\}$ are the elastic constants, $\alpha_{m}$ is the Madelung constant, $r_{0}$ is the nearest-neighbor separation distance, $e$ is the magnitude of the electron charge, $\mu$ is the reduced mass, $\omega_{\text {To }}$ is the transverse-optic frequency at the zone center, $v_{a}$ is the volume of the unit cell, $s$ is the Szigeti ef fective charge ratio, and $\epsilon_{0}$ and $\epsilon_{\infty}$ are the static and high-frequency dielectric constants, respectively. Equation (8) comes from the equilibrium condition. In addition, we use values of the polarizabilities $\alpha^{+}$and $\alpha^{-}$taken from the work of Jaswal and Sharma. ${ }^{14}$ The values of the constants which are not uniquely determined by Eqs. (5)-(10), are adjusted to obtain the best fits to the measured phonon dispersion curves. This involves varying of the relative values of $A^{+}$to $A^{-}, B^{+}$to $B^{-}, C^{+}$to $C^{-}$, and $\gamma_{+}$to $\gamma_{-}$.

The calculated dispersion curves along symmetry directions in the first Brillouin zone are shown in Figs. 1-4 for the four alkali fluorides. The curves for NaF have been presented earlier. ${ }^{6}$ Also shown are experimental points obtained by neutron

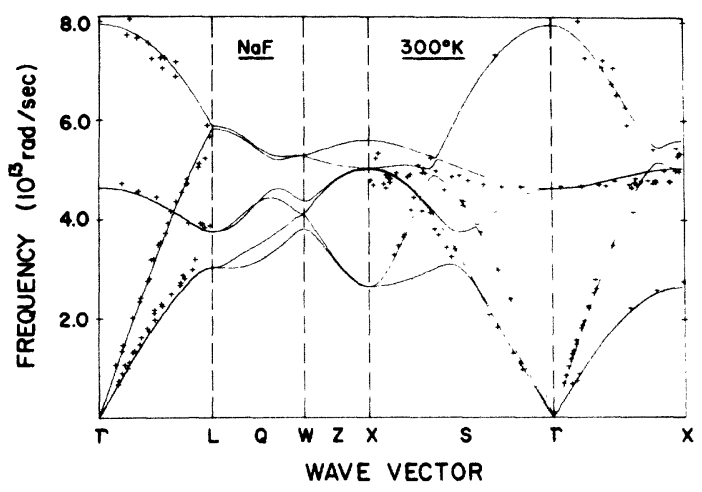

FIG. 1. Room-temperature phonon dispersion curves of $\mathrm{NaF}$ in the symmetry directions. The smooth curves are the calculated results based on a deformation dipole model and crosses are the neutron-scattering results from Ref. 15. 


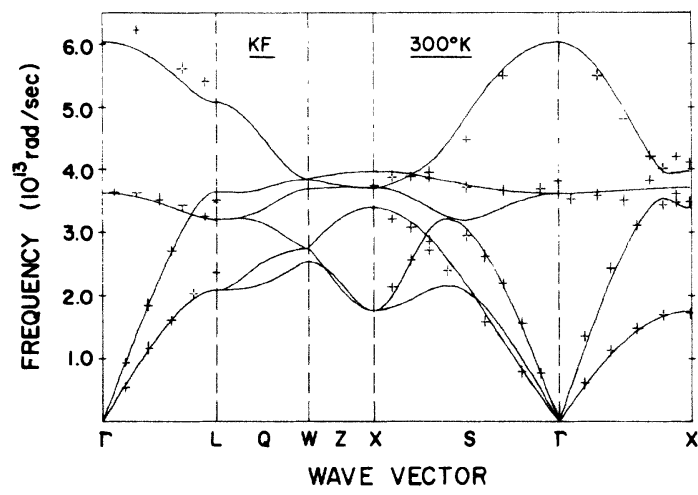

FIG. 2. Room-temperature phonon dispersion curves of $\mathrm{KF}$ in the symmetry directions. The smooth curves are the calculated results based on a deformation dipole model and the crosses are the neutron scattering results from Ref. 16.

diffraction by Buyers for NaF, ${ }^{15}$ Buhrer for KF, ${ }^{16}$ and Raunio and Rolandson for $\mathrm{RbF}^{17}$ and $\mathrm{CsF} .{ }^{18}$ The symmetry-point labels on the horizontal axes of Figs. 1-4 follow the standard notation introduced by Bouckaert, Smoluchowski, and Wigner. ${ }^{19}$ In Table I we present the experimental data used in obtaining the dispersion curves and in Table II we show the corresponding constants.

\section{PHONON POLARIZABILITY}

Following the theory of Born and Bradburn, ${ }^{1}$ the phonon polarizability $P_{\alpha \beta}\left(\vec{q} \mid j j^{\prime}\right)$ can be written in terms of real-space quantities as

$$
\begin{aligned}
P_{\alpha \beta}\left(\overrightarrow{\mathrm{q}} \mid j j^{\prime}\right)= & \sum_{\gamma \delta} \sum_{l \kappa \kappa^{\prime}} P_{\alpha \beta \gamma \delta}\left(l \mid \kappa \kappa^{\prime}\right)\left(m_{\kappa} m_{\kappa^{\prime}}\right)^{-1 / 2} \\
& \times \sigma_{\gamma}(\kappa \mid \overrightarrow{\mathrm{q}} j) \sigma_{5}\left(\kappa^{\prime} \mid-\overrightarrow{\mathrm{q}} j^{\prime}\right) e^{2 \mathbf{r} i \overrightarrow{\mathbf{q}} \cdot \overrightarrow{\mathrm{a}}\left(l \mid \kappa \kappa^{\prime}\right)} .
\end{aligned}
$$

Here, $m_{\kappa}$ is the mass of the $\kappa$-type ion, and $\vec{a}\left(l \mid \kappa \kappa^{\prime}\right)$ is the vector distance between the $\kappa$-type ion in the origin unit cell and the $\kappa^{\prime}$-type ion in the

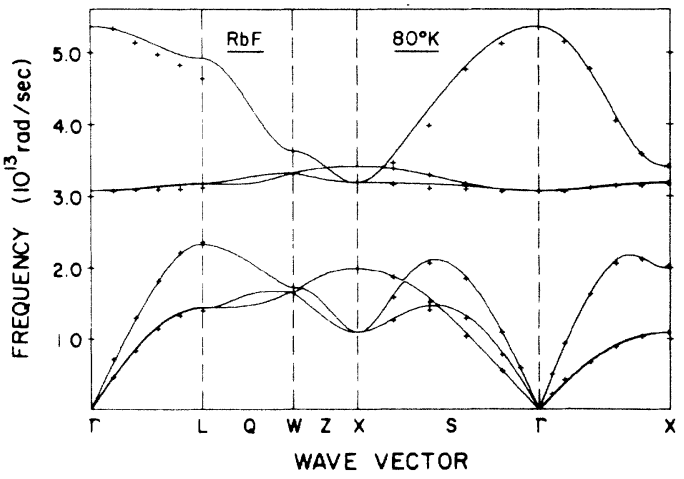

FIG. 3. Phonon dispersion curves at $80^{\circ} \mathrm{K}$ of $\mathrm{RbF}$ in the symmetry directions. The smooth curves are the calculated results based on a deformation dipole model and the crosses are the neutron scattering results from Ref. 17.

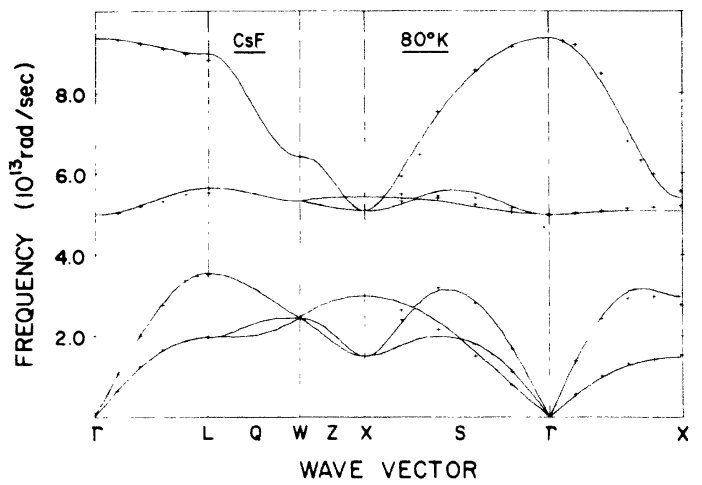

FIG. 4. Phonon dispersion curves at $80^{\circ} \mathrm{K}$ of $\mathrm{CsF}$ in the symmetry directions. The smooth curves are the calculated results based on a deformation dipole model and the crosses are the neutron scattering results from Ref. 18.

$l$ unit cell. The coefficients $P_{\alpha \beta \gamma \sigma}\left(l \mid \kappa \kappa^{\prime}\right)$ are called the polarizability coefficients and are formally given by

$$
P_{\alpha \beta \gamma \delta}\left(l \mid \kappa \kappa^{\prime}\right)=\left.\frac{\partial^{2} P_{\alpha \beta}}{\partial u_{\gamma}(0 \kappa) \partial u_{\sigma}\left(l \kappa^{\prime}\right)}\right|_{0},
$$

where $u_{\alpha}(l \kappa)$ is the $\alpha$-component of displacement from equilibrium of the $l \kappa$ ion, and the derivative is evaluated with all ions in their equilibrium con-

TABLE I. Input data for the lattice dynamic calculation for the four crystals.

\begin{tabular}{lllll}
\hline \multicolumn{1}{c}{ Parameter } & NaF & \multicolumn{1}{c}{ KF } & \multicolumn{1}{c}{ RbF } & CsF \\
\hline$c_{11}\left(10^{11} \mathrm{dyn} / \mathrm{cm}^{2}\right)$ & $9.71^{\mathrm{a}}$ & $6.48^{\mathrm{b}}$ & $6.7^{\mathrm{c}}$ & $5.27^{\mathrm{d}}$ \\
$c_{12}\left(10^{11} \mathrm{dyn} / \mathrm{cm}^{2}\right)$ & $2.43^{\mathrm{a}}$ & $1.70^{\mathrm{c}}$ & $1.2^{\mathrm{c}}$ & $1.42^{\mathrm{d}}$ \\
$c_{44}\left(10^{11} \mathrm{dyn} / \mathrm{cm}^{2}\right)$ & $2.80^{\mathrm{a}}$ & $1.55^{\mathrm{c}}$ & $1.1^{\mathrm{c}}$ & $0.81^{\mathrm{d}}$ \\
$r_{0}(\AA)$ & $2.31^{\mathrm{e}}$ & $2.674^{\mathrm{e}}$ & $2.815^{\mathrm{e}}$ & $3.004^{\mathrm{e}}$ \\
$s$ & $0.827^{\mathrm{f}}$ & $0.857^{\mathrm{g}}$ & $0.93^{\mathrm{h}}$ & $0.953^{\mathrm{g}}$ \\
$\alpha_{+}\left(10^{-24} \mathrm{~cm}^{-3}\right)^{1}$ & 0.285 & 1.149 & 1.679 & 2.740 \\
$\alpha_{-}\left(10^{-24} \mathrm{~cm}^{-3}\right)^{\mathrm{l}}$ & 0.876 & 0.876 & 0.858 & 0.858 \\
$\omega_{\text {TO }}\left(10^{13} \mathrm{sec}^{-1}\right)$ & $4.64^{\mathrm{d}}$ & $3.616^{\mathrm{k}}$ & $3.07^{1}$ & $2.487^{\mathrm{d}}$ \\
\hline \hline
\end{tabular}

${ }^{2} \mathrm{C}$. L. Anderson, Physical Acoustics, edited by W. P. Mason (Academic, New York, 1965), Vol. III, Part B.

${ }^{\mathrm{b} J}$. Marshall and R. F. Miller, J. Appl. Phys. $\underline{38}$, 4749 (1968).

cAdjusted.

dS. Rolandson, Phys. Stat. Solidi (B) $\underline{52}, 643$ (1972).

M. P. Tosi, Solid State Phys. 16, 1 (1964).

${ }^{1}$ A. M. Karo and J. R. Hardy, Phys. Rev. 181, 1272 (1969).

${ }^{8} \mathrm{~S}$. L. Cunningham, Ref. 13.

${ }^{\mathrm{n}} \mathrm{G}$. Raunio and S. Rolandson, Ref. 17 .

${ }^{1}$ S. S. Jaswal and T. P. Sharma, Ref. 14.

${ }^{\mathrm{S}} \mathrm{M}$. Born and K. Huang, Dynamical Theory of Crystal

Lattices (Clarendon, Oxford, England, 1954).

${ }^{k}$ C. M. Randall, R. M. Fuller, and D. J. Montgomery Solid State Commun. 2, 273 (1964).

${ }^{1}$ G. Raunio and S. Rolandson, Phys. Rev. B $\underline{2}, 2098$ (1970). 
TABLE II. Final parameters for the deformation dipole model.

\begin{tabular}{lcccc}
\hline \hline Parameter & $\mathrm{NaF}$ & $\mathrm{KF}$ & $\mathrm{RbF}$ & $\mathrm{CsF}$ \\
\hline$A$ & 9.912 & 10.85 & 12.39 & 12.00 \\
$B^{+}$ & -1.182 & -0.99 & -1.356 & -0.4946 \\
$A^{+}$ & 0.0 & 0.0 & -0.03809 & 0.8955 \\
$B^{+}$ & 0.0 & 0.0 & 0.04775 & -0.3352 \\
$A^{-}$ & -0.007185 & 0.1416 & -0.03809 & 0.0 \\
$B^{-}$ & 0.008744 & -0.08751 & 0.04775 & 0.0 \\
$C^{+}$ & 0.0 & -0.06646 & -0.02725 & -0.2156 \\
$C^{-}$ & 0.0 & 0.0 & -0.02725 & -0.2156 \\
$\gamma_{+}\left(10^{-11}\right)$ & 0.0 & 0.0 & 0.0 & -0.05066 \\
$\gamma_{+}^{\prime}\left(10^{-11}\right)$ & 0.0 & 0.0 & 0.0 & 1.229 \\
$\gamma_{-}^{-}\left(10^{-11}\right)$ & 0.6507 & 0.3852 & 0.2353 & 0.0 \\
$\gamma_{-}^{\prime}\left(10^{-11}\right)$ & -5.454 & -4.203 & -2.151 & 0.0 \\
\hline \hline
\end{tabular}

figuration. The polarizability coefficient $P_{\alpha \beta \gamma \delta}\left(l \kappa \kappa^{\prime}\right)$ is assumed to be symmetric in the first two indices; an assumption which is valid as long as the frequency shifts are not too large compared to the frequency of the incident light. ${ }^{20}$ Thus, for a single choice of two atoms $(0 \kappa)$ and $\left(l \kappa^{\prime}\right)$, there are $6 \times 9=54$ possible subscript combinations for the polarizability coefficients. This large number is reduced somewhat by the symmetry of the crystal and the following assumption. We assume that the size of the coefficients decreases as the distance between the two atoms under consideration increases. In particular, if the two atoms are farther apart than second neighbors, the polarizability coefficients are assumed to be zero.

In Fig. 5, we show the configuration of first and second neighbors in the rocksalt structure. If the polarizability coefficients are known for a single first-neighbor bond and for a single second-neighbor bond of both the ++ and -- type, then the polarizability coefficients for all other first-and second-neighbor bonds can be determined from symmetry. For the case of first-neighbor bonds, the three permutations of the coordinate axes together with the inversion operation interrelate the coefficients for all six first neighbors. For the case of second-neighbor bonds, the above operations coupled with reflection in the $x-y, y-z$, and $z-x$ planes interrelate the polarizability coefficients for all twelve second neighbors. The coefficients for a given first-neighbor bond, second-neighbor ++ bond, and second-neighbor -- bond, however, are not related.

The 54 possible coefficients for specific firstand second-neighbor bonds are not all independent. From group-theoretic arguments, the number of independent coefficients for a given bond is equal to the number of times the totally symmetric irreducible representation occurs in the reducible representation of the transformation matrix of the 54 polarizability coefficients. ${ }^{21}$ This can be determined by the equation

$$
m_{A}=\frac{1}{g} \sum_{j} h_{j} \chi_{j}(R),
$$

where $m_{A}$ is the number of times the $A_{1}$ (totally symmetric) irreducible representation occurs, $g$ is the order of the group, $h_{f}$ is the order of the $j$ th class, the sum is over all the classes of the group, and $\chi_{j}(R)$ is the character (trace) of the reducible representation for the symmetry operation $R$ which must be a member of the class $j$. This character is given by ${ }^{13}$

$$
\chi_{j}(R)=\left(4 \cos ^{2} \theta \pm 2 \cos \theta\right)\left(4 \cos ^{2} \theta \pm 4 \cos \theta+1\right),
$$

where $\theta$ is the angle of rotation associated with the symmetry operation $R$ and the + or - sign is chosen depending upon whether $R$ is a pure rotation or a rotation - reflection. Equation (15) results from the fact that the polarizability coefficient tensor relates a symmetric tensor of second-rank $P_{\alpha \beta}$ to an unsymmetric tensor of second-rank $u_{\gamma} u_{6}$. Thus its character is equal to the product of the character of a symmetric tensor and the character of an unsymmetric tensor.

For the first-neighbor bond in the [100] direction, the relevant group is $C_{4 v}(4 \mathrm{~mm})$. This group has 8 symmetry operations divided into the following five classes: (i) $E$, identity; (ii) $C_{2}$, rotation by $\pi$ around $x$ axis; (iii) $2 C_{4}$, rotation by $\pm \frac{1}{2} \pi$ around $x$ axis; (iv) $2 \sigma_{v^{\prime}}$, reflection in the two planes containing the $x$ axis and tilted at $\pm \frac{1}{4} \pi$ with respect to the $y$ axis. The use of Eqs. (14) and (15) leads to the result that there are only eight independent polarizability coefficients for first-neighbor bonds.

For the second-neighbor bond in the [110] direction, the relevant group is $C_{2}^{v}(m m 2)$. This group has 4 symmetry operations each in a separate class as follows: (i) $E$, identity; (ii) $C_{2}$, rotation by $\pi$ about the bond; (iii) $\sigma_{v^{\prime}}$, reflection about the plane

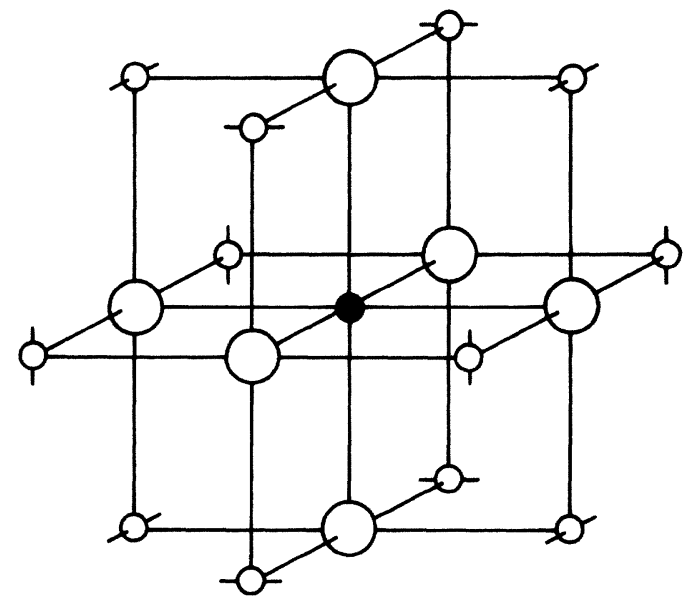

FIG. 5. First-neighbor (large circles) and secondneighbor (small circles) ionic configuration for the rocksalt structure. 
containing the $z$ axis and the bond; (iv) $\sigma_{d}$, reflection about the $x-y$ plane. Again using Eqs. (14) and (15), we find that there are 15 independent polarizability coefficients for each type (++ or - -) of secondneighbor bond.

The explicit coefficients that are allowed by group theory can be determined by the method of Fumi. ${ }^{21}$ In this method, the symmetry operations associated with each bond are applied to the subscripts of the polarizability coefficient tensor. The eight (15) sets of subscripts that are invariant under the symmetry operations for the first- (second-) neighbor bond are the independent elements.

The fact that 38 independent coefficients are allowed by group theory for the first - and secondneighbor bonds is unfortunate. At present there is no existing microscopic theory which can be used to predict the relative sizes of these terms. Consequently, all of these coefficients must be considered as adjustable parameters in the calculation unless limited by some additional assumptions.

In the previous calculations of the second-order Raman intensity, a wide range of different assumptions have been used to reduce the number of parameters. Born and Bradburn ${ }^{1}$ used a form of the phonon eigenvector which they assumed to be good for all phonons in the Brillouin zone. This form led to the fact that the eight first-neighbor coefficients occurred in only three different linear combinations in the expressions for the phonon polarizability. Hence, their calculation only required three adjustable parameters. Smith $^{2}$ used a similar procedure for diamond.

Cowley $^{3}$ introduced a different and very interesting point of view. He introduced anharmonic force constants into a shell model lattice dynamic calculation and related the phonon polarizability to these anharmonic constants. In this way he was able to reduce the number of parameters to two. How ever, the calculations were performed before detailed Raman data were available, and the comparison with experiment has since been shown to be poor. More recent calculations by Bruce and Cow1 ley ${ }^{7}$ extending the earlier approach employ the anharmonicity in the interatomic forces. This approach was applied to $\mathrm{KBr}$ and predicts reasonably well the positions of the main spectral peaks but does not reproduce the over-all shape of the spectrum.

A third approach has been used by Hardy, Karo, Morrison, Sennett, and Russell. ${ }^{5}$ They follow the Born-Bradburn approach and obtain expressions containing the eight first-neighbor parameters. Then they assume that the polarizability is affected only by central displacements of the nearest neighbors (i.e., changes in bond length). This reduces the number of parameters to three and results in the spectrum with $T_{2 \varepsilon}$ symmetry being identically zero. Their calculations were compared with a microdensitometer trace for NaF. More recently, Cunningham, Hardy, and $\mathrm{Hass}^{6}$ attempted to improve the calculation for NaF by introducing second-neighbor parameters and a slight variation of the central displacement assumption. Their assumption reduced the 38 first- and second-neighbor parameters to 11 which were further reduced to seven by neglecting the second-neighbor coefficients for ++ bonds. Their results, however, are incorrect due to a computer prog ramming error.

$\mathrm{Krauzman}^{9}$ has performed a calculation for $\mathrm{KBr}$ using only first-neighbor polarizability coefficients. In his calculation, the eight coefficients are reduced to two by assuming that the five coef ficients for the $A_{1 g}$ and $E_{g}$ spectra are all equal (this results in the calculated $E_{g}$ spectrum vanishing), and that only one of the three coefficients for the $T_{2 g}$ spectrum is nonzero (this second assumption is unclear from the paper). The assumptions made by Krauzman are ones of convenience and are not based on any microscopic model. The calculation reproduces some of the experimental peaks, but not the over-all shape of the spectra.

Finally, Pasternak, Cohen, and Gilat ${ }^{8}$ have performed a calculation for $\mathrm{MgO}$ using both first - and second-neighbor coefficients. They reduce the number of coefficients by introducing two assumptions: First, they assume that the polarizability derivative only depends on the projection of the ionic displacement on the direction of the light polarization. For the term $P_{\alpha \beta \gamma \delta}\left(l \mid \kappa \kappa^{\prime}\right)$ this makes $\alpha=\gamma$ and $\beta=\delta$ or $\alpha=\delta$ and $\beta=\gamma$ and makes these two types of terms equal for a given choice of $\alpha$ and $\beta$. This assumption reduces the number of coefficients from 38 to 12 , four for each type of neighbor bond. Second, they assume that the coefficients are independent of $l$ which reduces the number to six, three for the $i_{1111}(\omega)$ spectrum and three for the $i_{1212}(\omega)$ spectrum. In comparing the calculation with experiment, only one coefficient for each spectrum was used.

With the possible exception of Cowley's work, each assumption introduced in the above-mentioned work has no microscopic basis and is at best only plausible. In a recent paper, Jaswal, Wolfram, and Sharma ${ }^{10}$ have shown.that there is no a priori reason for most of the assumptions mentioned above. They determine the various parameters by making detailed comparisons between calculated and experimental spectra. In this way they obtained very good agreement between experimental and theoretical spectra for both $\mathrm{LiH}$ and $\mathrm{LiD}$. In the present paper, we will use the same approach as that in Ref. 10. A discussion of the calculations and results follows the section on the experimental spectra. 
TABLE III. Relation between the experimental spectra and the three spectral components in the two schemes.

\begin{tabular}{clc}
\hline \hline Experimental $^{\mathrm{a}}$ & \multicolumn{2}{c}{ Spectral components } \\
\hline $1 x(y y) z$ & $i_{1111}$ & $\frac{1}{3}\left[I\left(A_{1 z}\right)+I\left(E_{z}\right)\right]$ \\
$2 x(y x) z$ & $i_{1212}$ & $I\left(T_{2 z}\right)$ \\
$3 a(b a) z$ & $\frac{1}{2}\left(i_{1111}-i_{1122}\right)$ & $\frac{1}{4} I\left(E_{z}\right)$ \\
\hline \hline
\end{tabular}

$2 x, y, z$ refer to crystal body axes [100], [010], [001]. $a, b, z$ refer to directions [110], [110], [001].

${ }^{b}$ The designation of the numerical prefactor for the $E_{\boldsymbol{g}}$ spectral component may vary with the author.

\section{EXPERIMENTAL SPECTRA}

The terms $i_{\alpha \beta \gamma \delta}(\omega)$ in Eq. (2) have the same symmetry properties as the elastic constants. Thus, for the rocksalt structure there are only three independent terms which we will label $i_{1111}(\omega), i_{1122}(\omega)$, and $i_{1212}(\omega)$. Any measured spectrum will be some linear combination of only these three spectral components.

Similarly, it can be shown that if the polarizability tensor $P_{\alpha \beta}$ is assumed to be symmetric, there are only three group-theoretic irreducible representations appropriate for the terms $i_{\alpha \beta \gamma 6}(\omega)$. These are the $A_{1 g}, E_{g}$, and $T_{2 g}$ irreducible representations. Thus, any measured spectrum can also be expressed as a linear combination of the three group-theoretic spectra.

Unfortunately, all three of the independent spectral components in either of the above two schemes cannot be measured separately. In the first scheme, $i_{1122}$ cannot be measured separately, and in the second scheme, the spectra with $A_{18}$ symmetry cannot be measured separately. The other two spectral components in each scheme can be measured separately but, due to the fact that each measured spectrum has an unknown background,

TABLE IV. Subscripts of the nonzero first- and second-neighbor polarizability coefficients and their numerical values [normalized with respect to the $P_{1111}(01+-)$ value] for the spectra for the four crystals containing $A_{1,}$ and $E_{8}$ symmetry components.

\begin{tabular}{lcccr}
\hline \multicolumn{1}{c}{ Subscripts } & NaF & KF & RbF & CsF \\
\hline First neighbor & & & & \\
1111 & 1.0 & 1.0 & 1.0 & 1.0 \\
$2222=3333$ & 0.0 & -0.11 & -0.343 & -0.28 \\
$2233=3322$ & -0.191 & -0.15 & -0.09 & -0.08 \\
$1122=1133$ & -0.0497 & -0.05 & -0.045 & -0.04 \\
$2211=3311$ & 0.485 & 0.25 & 0.098 & 0.12 \\
Second neighbor & & & & \\
$1111=2222(++)$ & 0.0 & 0.0 & 0.0 & 0.0 \\
$1111=2222(--)$ & 0.0 & 0.182 & 0.0 & 0.0 \\
\hline \hline
\end{tabular}

TABLE V. Subscripts of the nonzero first- and second-neighbor polarizability coefficients and their numerical values [normalized with respect to the $R_{221}(0 \mid+-)$ value] for the $T_{2 \varepsilon}$ spectra for the four crystals.

\begin{tabular}{lrccc}
\hline \multicolumn{1}{c}{ Subscripts } & NaF & KF & RbF & CsF \\
\hline First neighbor & & & & \\
$1221=3131$ & 1.0 & 1.0 & 1.0 & 1.0 \\
$1212=3113$ & 0.6 & 0.682 & 0.143 & 0.13 \\
$2323=2332$ & -0.6 & -0.364 & -0.143 & -0.13 \\
Second neighbor & & & & \\
$1211=1222(++)$ & -0.2 & 0.0 & 0.0 & 0.0 \\
$1211=1222(--)$ & 0.8 & 0.455 & 0.571 & 0.0 \\
\hline \hline
\end{tabular}

extracting the third component can introduce undetermined error. Thus, in this paper, we will calculate the measured spectra rather than reduce the measured spectra to the three independent spectral components. In Table III we show the relation between the three measured spectra and the three spectral components in each scheme.

The three different spectra indicated in Table III were obtained at room temperature for each of the four alkali fluoride crystals $\mathrm{NaF}, \mathrm{KF}, \mathrm{RbF}$, and CsF. The apparatus used the $4880-\AA$ line of an argon-ion laser. The scattered light was collected at $90^{\circ}$ relative to the incident beam by a wide-angle lens $(f / 0.95)$ and analyzed by a Spex double mono-

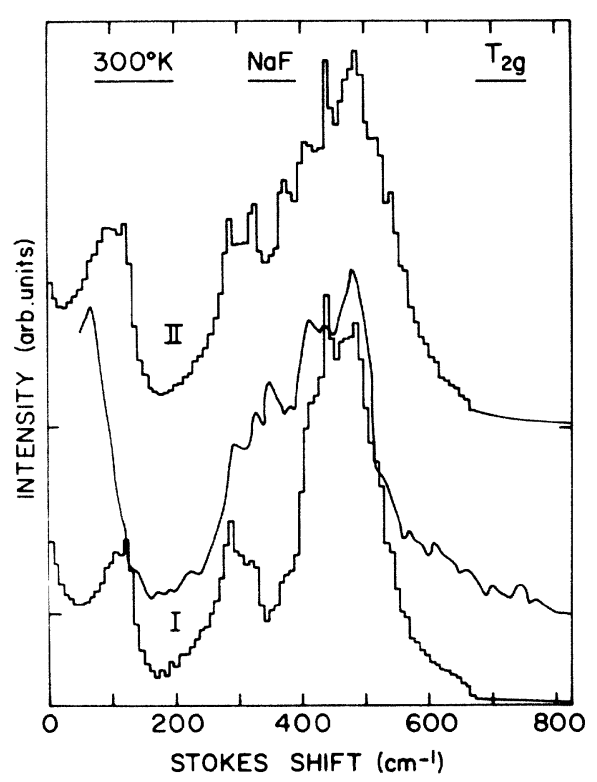

FIG. 6. Room-temperature $T_{2 g}$ spectra of NaF. The smooth curve is the experimental spectrum and histograms I and II are the calculated spectra in the nearestneighbor and next-nearest-neighbor polarizability approximations, respectively. 
TABLE VI. Calculated phonon frequencies $\left(\mathrm{cm}^{-1}\right)$ at various Brillouin-zone symmetry points for the four alkali fluorides.

\begin{tabular}{ccrrr}
\hline \hline Mode label & NaF & KF & RbF & CsF \\
\hline TO $(\Gamma)$ & 246 & 192 & 163 & 132 \\
LO $(\Gamma)$ & 422 & 320 & 285 & 248 \\
TA $(L)$ & 161 & 110 & 78 & 52 \\
LA $(L)$ & 310 & 193 & 124 & 94 \\
TO $(L)$ & 199 & 170 & 169 & 149 \\
LO $(L)$ & 313 & 270 & 262 & 238 \\
TA $(X)$ & 141 & 93 & 58 & 39 \\
LA $(X)$ & 266 & 180 & 106 & 79 \\
TO $(X)$ & 268 & 197 & 169 & 134 \\
LO $(X)$ & 298 & 210 & 182 & 144 \\
\hline \hline
\end{tabular}

chromator coupled to a photon-counting photomultiplier.

The samples were of varying optical quality, ranging from excellent for $\mathrm{NaF}$ to poor for $\mathrm{RbF}$ and CsF. These last two crystals are hygroscopic which caused the faces to appear somewhat cloudy since the experiments were performed in air (the scattering volume being sampled was well within the bulk of the crystals). In addition, the crystals are soft which makes polishing difficult.

The spectra for the four crystals are presented in Figs. 6-21. In all cases, except for $\mathrm{RbF}$, a linear background has been subtracted from the

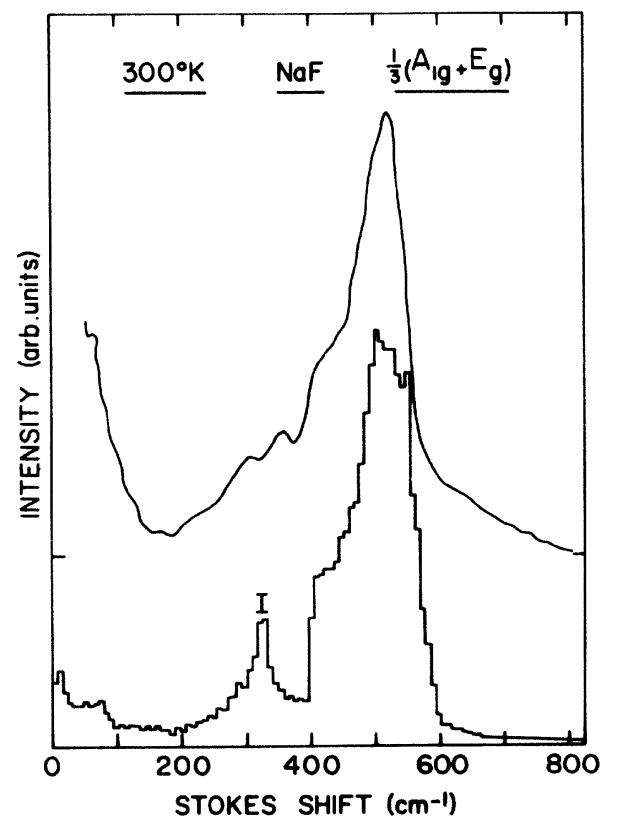

FIG. 7. Room-temperature $\frac{1}{3}\left(A_{1 g}+E_{g}\right)$ spectra of NaF. The smooth curve is the experimental spectrum and histogram I is the calculated spectrum in the nearestneighbor polarizability approximation.

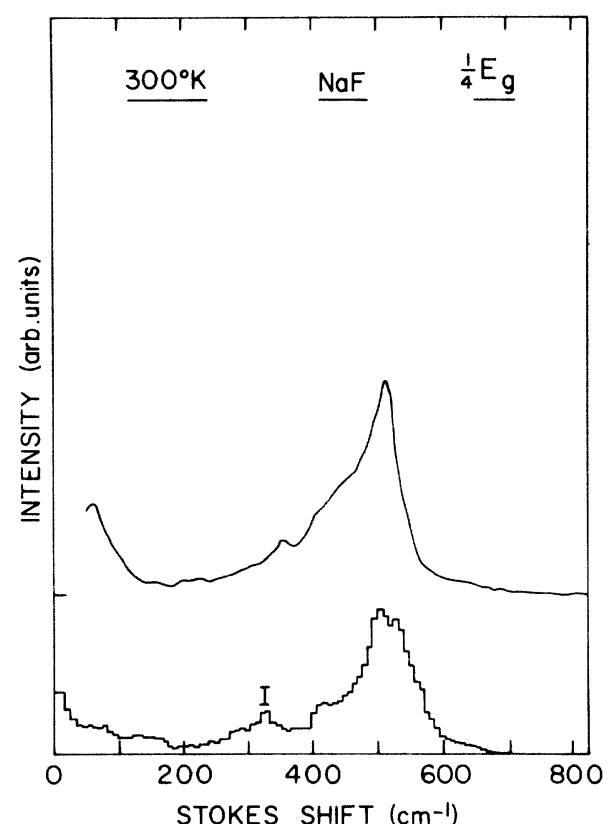

FIG. 8. Room-temperature $\frac{1}{4} E_{\boldsymbol{g}}$ spectra of NaF. The smooth curve is the experimental spectrum and histogram I is the calculated spectrum in the nearestneighbor polarizability approximation.

observed spectra to remove, as far as possible, the tail of the Rayleigh line. This procedure was not used for $\mathrm{RbF}$ due to the uncertainties in the raw data, which were largest for this crystal.

\section{NUMERICAL CALCULATIONS AND RESULTS}

We treat the 38 polarizability coefficients as variables in least-squares fits to each of the measured spectra. This gives us a trial set of pa-

TABLE VII. Contributions of two-phonon combinations from two Brillouin-zone symmetry points to the Raman active irreducible representations. The irreducible representation for the single phonon was determined from the symmetry of the eigenvector. Two-phonon symmetry was then obtained from Ref. 23.

\begin{tabular}{lcccccc}
\hline \hline & \multicolumn{3}{c}{$X$ Point } & \multicolumn{3}{c}{$L$ Point } \\
Phonon Combination & $A_{1 \boldsymbol{g}}$ & $E_{\boldsymbol{g}}$ & $T_{2 \boldsymbol{g}}$ & $A_{1 \boldsymbol{g}}$ & $E_{\boldsymbol{g}}$ & $T_{2 \boldsymbol{g}}$ \\
\hline $\pm 2 \mathrm{LO}$ & $X$ & $X$ &. & $X$ &. & $X$ \\
$\pm \mathrm{LO} \pm \mathrm{TO}$ &. &. & $X$ &. & $X$ & $X$ \\
$\pm \mathrm{LO} \pm \mathrm{LA}$ & $X$ & $X$ &. &. &. & $\cdot$ \\
$\pm \mathrm{LO} \pm \mathrm{TA}$ &. &. & $X$ &. &. &. \\
$\pm 2 \mathrm{TO}$ & $X$ & $X$ & $X$ & $X$ & $X$ & $X$ \\
$\pm \mathrm{TO} \pm \mathrm{LA}$ &. &. & $X$ &. &. &. \\
$\pm \mathrm{TO} \pm \mathrm{TA}$ & $X$ & $X$ & $X$ &. &. &. \\
$\pm 2 \mathrm{LA}$ & $X$ & $X$ &. & $X$ &. & $X$ \\
$\pm \mathrm{LA} \pm \mathrm{TA}$ &. &. & $X$ &. & $X$ & $X$ \\
$\pm 2 \mathrm{TA}$ & $X$ & $X$ & $X$ & $X$ & $X$ & $X$ \\
\hline \hline
\end{tabular}




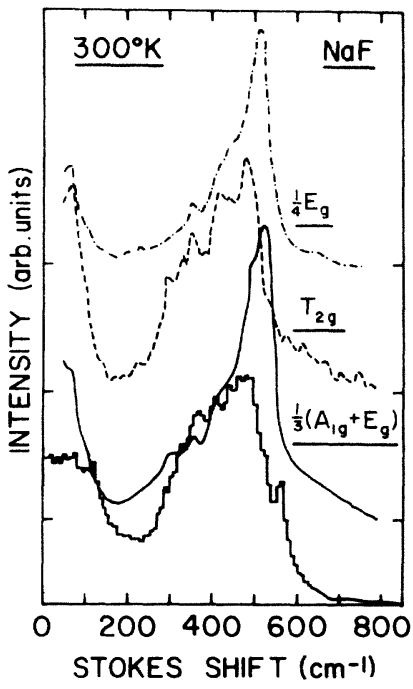

FIG. 9. Room-temperature experimental Raman spectra (smooth lines) and the temperature-weighted twophonon density-of-states histogram for NaF. The scales for the $T_{2 g}$ and $\frac{1}{4} E_{g}$ spectra have been amplified ten times and $\frac{20}{3}$ times, respectively.

rameters for each spectrum of a particular crystal. These are then further refined by examining in detail the contributions of individual parameters to each spectrum and making adjustments to improve the fit to specific features. The values of the nonzero polarizability coefficients which re-

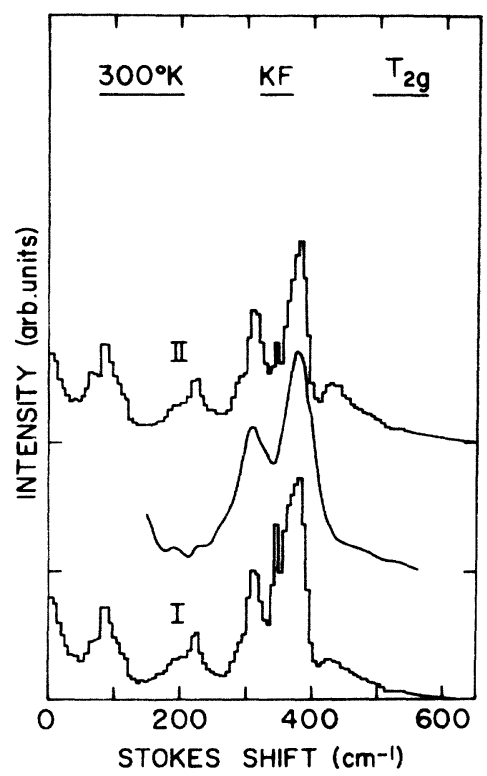

FIG. 10. Room-temperature $T_{2 g}$ spectra of KF. The smooth curve is the experimental spectrum and histograms I and II are the calculated spectra in the nearestneighbor and next-nearest-neighbor polarizability approximations, respectively.

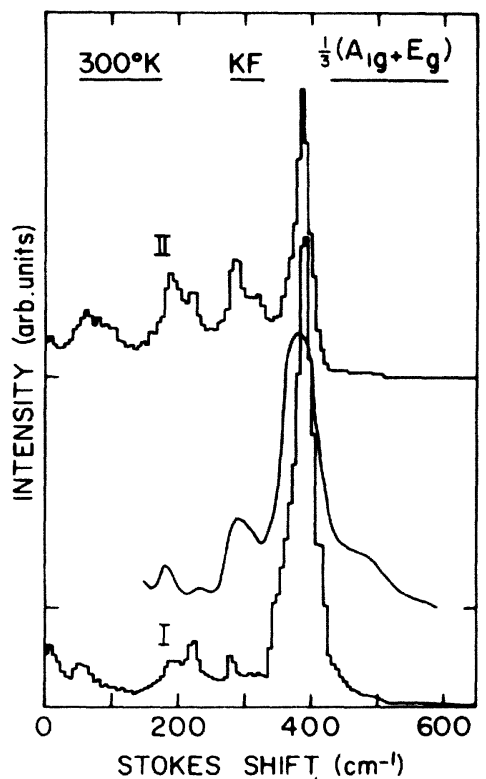

FIG. 11. Room-temperature $\frac{1}{3}\left(A_{1 g}+E_{g}\right)$ spectra of KF. The smooth curve is the experimental spectrum and histograms I and II are the calculated spectra in the nearest-neighbor and next-nearest-neighbor polarizability approximations, respectively.

sult from this fitting procedure are given in Tables IV and V.

Fortunately we can obtain adequate agreement in all cases if we retain only the first-neighbor coef-

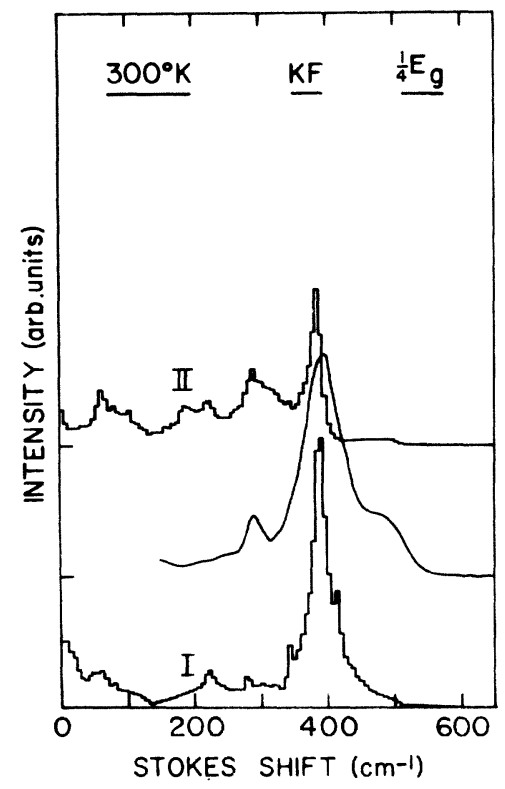

FIG. 12. Room-temperature $\frac{1}{4} E_{g}$ spectra of KF. The smooth curve is the experimental spectrum and histograms I and II are the calculated spectra in the nearest-neighbor and next-nearest-neighbor polarizability approximations, respectively. 
ficients together with only one or two secondneighbor coefficients, the last being adjusted in a specific manner in any given case. Thus in our initial fits we have only four disposable parameters to fit the $A_{1 g}$ and $E_{8}$ spectra and only two for the $T_{28}$ spectra. This is so because we are not calculating absolute intensities but only relative magnitudes.

On this basis we feel that the numerical values of the parameters have a definite physical meaning; specifically one can argue that the truncation of the sum in Eq. (12) at the second-neighbor sites is valid. As a consequence, it should be possible to demonstrate by a detailed quantum-mechanical calculation that the polarizability of a given ion is mainly influenced by first-neighbor and to a lesser extent by second-neighbor displacements. We shall now proceed to discuss each crystal in the sequence separately.

\section{A. Sodium fluoride}

In Figs. 6-8, we show the three spectral components (c.f. Table III) for NaF, the smooth curves being the experimental results and the histograms representing the computed spectra. Here, and elsewhere, the histograms labeled I have been computed using only first-neighbor coefficients shown in Tables IV and V, while the histograms labeled II have been computed using both the firstand second-neighbor coefficients in Tables IV and V.

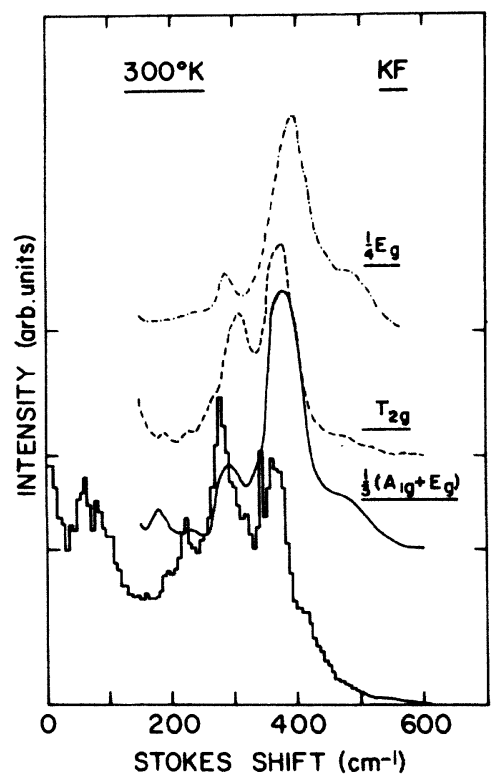

FIG. 13. Room-temperature experimental spectra (smooth lines) and the temperature-weighted-two-phonon density-of-states histogram for KF. The scales for the $T_{2 g}$ and $\frac{1}{4} E_{g}$ spectra have been amplified five times and two times, respectively.

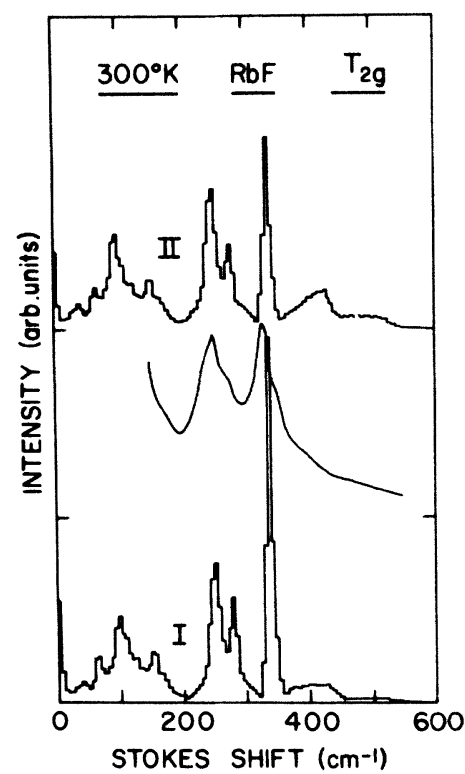

FIG. 14. Room-temperature $T_{2 g}$ spectra of RbF. The smooth curve is the experimental spectrum and histograms I and II are the calculated spectra in the nearestneighbor and next-nearest-neighbor polarizability approximations, respectively.

For the $T_{2 g}$ spectrum (Fig. 6), second-neighbor parameters are necessary to produce the peaks be tween 320 and $400 \mathrm{~cm}^{-1}$. For the other two spectra, the first-neighbor coefficients alone provide an adequate reproduction of the measured spectra. It should be remarked that the $\frac{1}{3}\left(A_{1 g}+E_{g}\right)$ spectrum (Fig. 7) shows two peaks between 300 and $385 \mathrm{~cm}^{-1}$

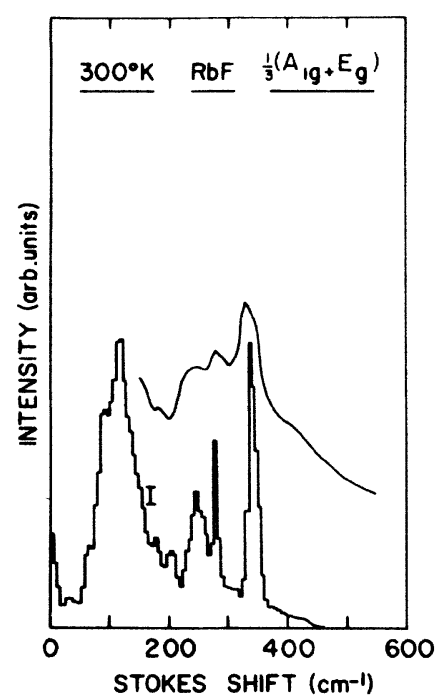

FIG. 15. Room-temperature $\frac{1}{3}\left(A_{1 g}+E_{g}\right)$ spectra of $\mathrm{RbF}$. The smooth curve is the experimental spectrum and histogram $I$ is the calculated spectrum in the nearestneighbor polarizability approximation. 


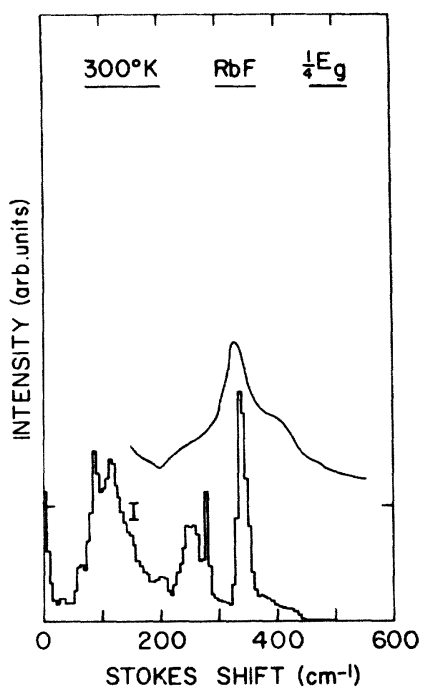

FIG. 16. Room-temperature $\frac{1}{4} E_{g}$ spectra of $\mathrm{RbF}$. The smooth curve is the experimental spectrum and histogram I is the calculated spectrum in the nearestneighbor polarizability approximation.

while data taken by Evans and Fitchen ${ }^{22}$ show only one peak in this region.

In Table VI we show the computed phonon frequencies for various symmetry points for all four crystals and in Table VII we show all possible combinations of these same symmetry-point phonons and indicate which are allowed or forbidden by group theory. While in no case is it possible to make detailed assignments of the peaks in the observed spectra, one can sometimes observe

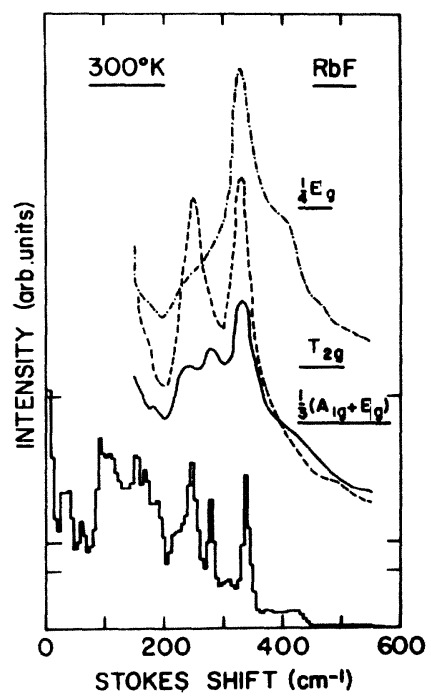

FIG. 17. Room-temperature experimental spectra (smooth lines) and the temperature-weighted-two-phonon density-of-states histogram for RbF. The scales for the $T_{2 g}$ and $\frac{1}{4} E_{g}$ spectra have been amplified four times.

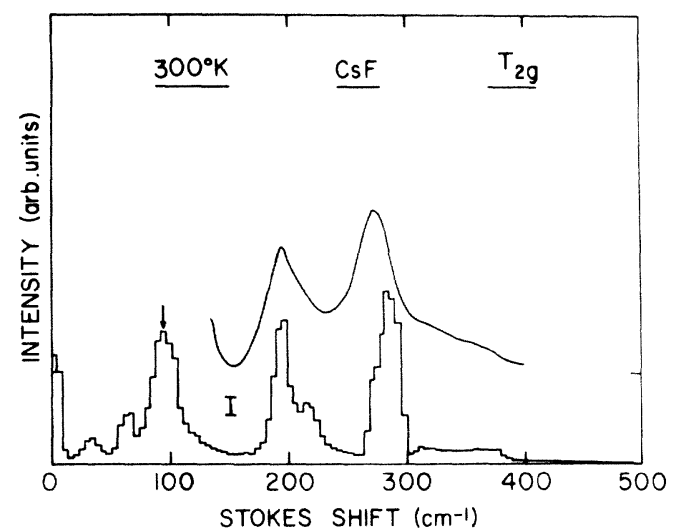

FIG. 18. Room-temperature $T_{2 g}$ spectra of CsF. The smooth curve is the experimental spectrum and histogram I is the calculated spectrum in the nearestneighbor polarizability approximation. The arrow at 92 $\mathrm{cm}^{-1}$ gives the position of the experimental difference band peak seen by Evans and Fitchen (Ref. 22).

that peaks are present which cainnot be assigned to symmetry combinations. Such a case is the $300-\mathrm{cm}^{-1}$ peak in the $\frac{1}{3}\left(A_{1 g}+E_{g}\right)$ spectrum of NaF (Fig. 7). The theoretical spectrum shows a single peak at $325 \mathrm{~cm}^{-1}$ and fails to reproduce the second peak at $385 \mathrm{~cm}^{-1}$, but a combination of TA and TO phonons from near the $L$ point could give a peak in this region of the spectrum.

In the case of the $\frac{1}{4} E_{g}$ spectrum (Fig. 8) we obtain a good fit except that once again, the theoretical and experimental peaks between 300 and 400 $\mathrm{cm}^{-1}$ do not coincide.

Finally, in Fig. 9 we show all three experimental spectra compared with the histogram computed assuming that the Fourier transformed polarizability coefficients [Eq. (12)] are constant and equal (this is the temperature weighted two-phonon density of states). One can see that there is little correlation between this and any of the observed spectra both as regards peak positions and relative intensities. Thus, it is very important to include the phonon polarizability in any calculation that is to be compared with experiment.

\section{B. Potassium fluoride}

In Figs. 10-13 we show the same results for KF as those presented for NaF. This compound was the most difficult of the four in terms of obtaining agreement between theory and experiment. It was essential to include second-neighbor polarizability coefficients when calculating all three spectra. Specifically, the second-neighbor coefficients for the negative ions were used to supress the peak at $344 \mathrm{~cm}^{-1}$ and enhance that at $425 \mathrm{~cm}^{-1}$ in the $T_{2 \mathrm{~g}}$ spectrum (Fig. 10). In the other two spectra, they were needed to lower the relative intensity of the main peak while enhacing the intensity of the sub- 


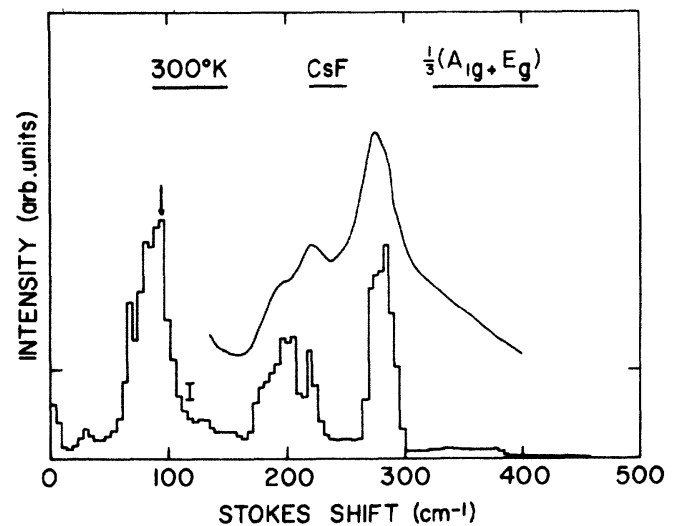

FIG. 19. Room-temperature $\frac{1}{3}\left(A_{1 g}+E_{g}\right)$ spectra of CsF. The smooth curve is the experimental spectrum and histogram I is the calculated spectrum in the nearestneighbor polarizability approximation. The arrow at 92 $\mathrm{cm}^{-1}$ gives the position of the experimental difference band peak seen by Evans and Fitchen (Ref. 22).

\section{sidiary features.}

The over-all agreement between theory and experiment is satisfactory except that the observed $\frac{1}{4} E_{g}$ spectrum (Fig. 12) appears to have a high-frequency shoulder which the theory fails to reproduce.

In Fig. 13 we compare all three observed spectra with the calculated spectrum obtained assuming constant coupling to all phonon pairs. As was the case for $\mathrm{NaF}$, there is little correlation with the experimental spectra.

\section{Rubidium fluoride and cesium fluoride}

For these crystals the experimental data are worse than those for the other two fluorides, owing to the poor optical quality of the samples. The observed spectra are superposed on a broad and ill-

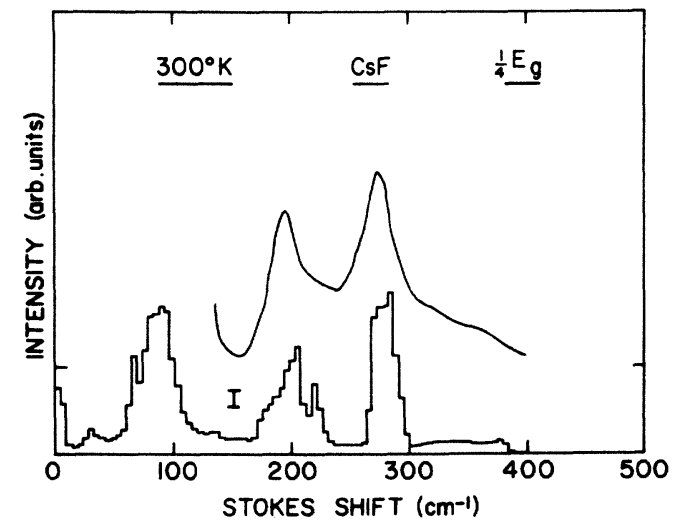

FIG. 20. Room-temperature $\frac{1}{4} E_{g}$ spectra of CsF. The smooth curve is the experimental spectrum and histogram $I$ is the calculated spectrum in the nearestneighbor polarizability approximations.

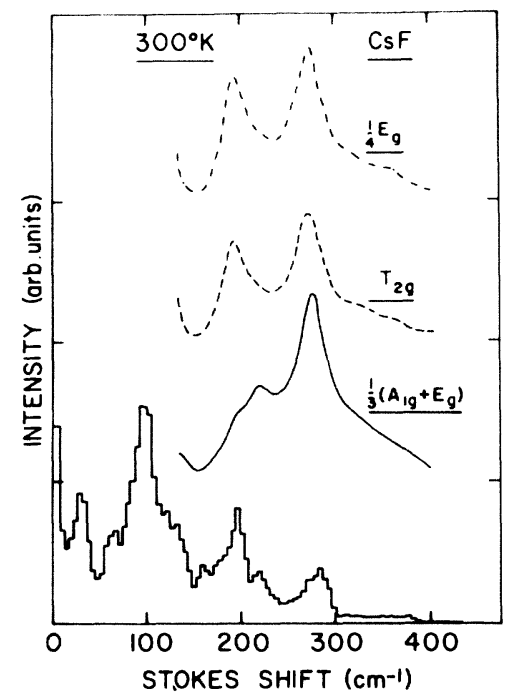

FIG. 21 Room-temperature experimental spectra (smooth lines) and the temperature-weighted-two-phonon density-of-states histogram for CsF. The scales for $T_{2 g}$ and $\frac{1}{4} E_{g}$ spectra have been amplified two times.

determined Rayleigh background and it is impossible to study the low-frequency region where the difference bands should lie.

The spectra are presented in the same way as before; Figs. 14-17 show theoretical and experimental data for RbF and Figs. 18-21 show the corresponding data for $\mathrm{CsF}$. In the absence of room temperature dispersion curves, we have felt it best to use the eigendata calculated using $80^{\circ} \mathrm{K}$ input parameters.

Except for the $T_{2 g}$ spectrum of RbF (Fig. 14), all the spectra can be reproduced adequately if we employ only first-neighbor polarizability coefficients. However, the observed $\frac{1}{4} E_{\mathrm{g}}$ spectrum of $\mathrm{RbF}$ (Fig. 16) shows only a shoulder where the computed spectrum shows a double peak.

It is fair to say that we have a better fit for CsF than for RbF, a contention which is supported by the presence of a difference band at $90 \mathrm{~cm}^{-1}$ in the computed CsF spectra which has been observed by Evans and Fitchen. ${ }^{22}$ Undoubtedly we could refine our calculations further given more clearly resolved experimental spectra. After this work was completed, experimental Raman data for RbF by Hayes and Rieder ${ }^{24}$ appeared. Our spectra agree quite well with their room-temperature measurements.

\section{CONCLUSION}

In this paper we have presented theoretical and experimental second-order Raman spectra for $\mathrm{NaF}, \mathrm{KF}, \mathrm{RbF}$, and CsF. The experimental spectra reveal that for the first two materials the $T_{2 \mathrm{~s}}$ spectrum is relatively weak, but that for the last 
two its strength is comparable with that of the other spectral components. Moreover, the shapes of the spectra for the first two fluorides are qualitatively different from those for the last two. The main reason for this is presumably the increased split ting between the optic and acoustic branches of the spectra for the heavier fluorides. This results in (acoustic + acoustic) and (acoustic + optic) combi nations being significantly shifted in frequency with respect to (optic + optic) combinations whereas, for the lighter fluorides, the combinations of all types strongly overlap.

As regards the theory, one can make the following general remarks:

(a) It is essential to allow for the dependence of the transformed polarizabilities on the phonon wave vectors and eigenvectors. Without this there is little resemblance between the theoretical and experimental spectra. This point was made previously for the cases of $\mathrm{NaF}$ and $\mathrm{KF}$ and is equally true for the other two fluorides.

(b) We can fit all spectra reasonably well using relatively few parameters. Moreover, there is a marked similarity between the relative magnitudes of the polarizability parameters for all four crys- tals. These two facts are good evidence for the belief that the parameter values are physically meaningful and that the use of the technique of Born and Bradburn ${ }^{1}$ is successful.

(c) Our lattice dynamical model appears to give a good account of the measured dispersion curves. There is, however, a curious anomaly. Normally we locate the deformation dipoles at the centers of the negative ions. But in the case of CsF, it was found that a better fit could be obtained by locating them at the centers of the positive ions without altering their sign (see Table II). A possible explanation is that increasing overlap tends to transfer charge from the positive to the negative ion. How ever, there is no sign of any such tendancy for $\mathrm{RbF}$.

Evidently our calculations are capable of further refinement, particularly for RbF and CsF, but this is not worthwhile in the absence of more precise experimental data. In particular, this data should be taken at low temperatures where we expect more structure to become apparent since we sus pect that strong anharmonic effects are present for the heavier fluorides at $300^{\circ} \mathrm{K}$ and that these have obscured such structure.
*Work supported in part by the National Science Foundation under Departmental Development Grant No. GU 3163 .

†Part of this work was done while the author was at the Physics Department, University of California, Irvine, Calif. 92664 under the support of the Office of Naval Research, Contract No. N00014-69-A-0200-9003.

₹Supported by the Research Council of the Universtiy of Nebraska.

§Present address: Naval Research Laboratory, Code 6408, Washington, D. C. 20375.

${ }^{1}$ M. Born and M. Bradburn, Proc. R. Soc. A $\underline{188}, 161$ (1947).

${ }^{2}$ H. M. J. Smith, Phil. Trans. R. Soc. Lond. A 241 , 105 (1948).

${ }^{3}$ R. A. Cowley, Proc. Phys. Soc. Lond. 84,281 (1964).

${ }^{4}$ A. M. Karo, J. R. Hardy, and I. W. Morrison, J. Phys. 26, 668 (1965); J. R. Hardy and A. M. Karo, Phys. Rev. 141, 696 (1966); Phys. Rev. 160, 702 (1967); Phys. Rev. 168, 1054 (1968); J. Chem. Phys. 48,3173 (1968).

5J. R. Hardy, A. M. Karo, I. W. Morrison, C. T. Sennett, and J. P. Russell, Phys. Rev. 179, 837 (1969).

${ }^{6}$ S. L. Cunningham, J. R. Hardy, and M. Hass, Light Scattering in Solids, Proceedings of the Second International Conference, edited by M. Balkanski (Flammarion, Paris, 1971), p. 257.

${ }^{7}$ A. D. Bruce and R. A. Cowley, J. Phys. C $\underline{5}, 595$ (1972).

${ }^{8}$ A. Pasternak, E. Cohen, and G. Gilat, Solid State Commun. 12, 451 (1973). A more detailed discussion and application to $\mathrm{KBr}, \mathrm{NaCl}$, and $\mathrm{MgO}$ can be found in Phys. Rev. B 9 , 4584 (1974).

${ }^{9}$ M. Krauzman, Solid State Commun. 12, 157 (1973).

${ }^{10}$ S. S. Jaswal, G. Wolfram, and T. P. Sharma, J. Phys. Chem. Solids $\underline{35}, 571$ (1974).

${ }^{11}$ T. P. Sharma, R. D. Kirby, and S. S. Jaswal, Phys. Rev. B. $\underline{9}, 1971$ (1974).

${ }^{12}$ J. R. Hardy, Philos. Mag. $\underline{6}, 27$ (1961), Philos. Mag. 7, 315 (1962).

${ }^{13} \overline{\mathrm{S}}$. L. Cunningham, Ph.D. thesis (University of Nebraska, 1971) (unpublished).

${ }^{14}$ S. S. Jaswal and T. P. Sharma, J. Phys. Chem. Solids 34, 509 (1973).

${ }^{15}$ W. J. L. Buyers, Phys. Rev. 153, 923 (1967).

${ }^{16}$ W. Buhrer, Phys. Status. Solidi 41,789 (1970).

${ }^{17}$ G. Raunio and S. Rolandson, J. Phys. C 3 , 1013 (1970).

${ }^{18}$ G. Raunio and S. Rolandson, Phys. Status Solidi (B) $\underline{52}$, 6431 (1972).

${ }^{19}$ L. P. Bouckaert, R. Smoluchowski, and E. Wigner, Phys. Rev. 50, 58 (1936).

${ }^{20}$ R. Loudon, Proc. R. Soc. A 275, 218 (1963).

${ }^{21} \mathrm{~S}$. Bhagavantam and T. Venkatarayudu, Theory of Groups and Its Application to Physical Problems (Academic, New York, 1969).

${ }^{22}$ A. R. Evans and D. B. Fitchen, Solid State Commun. 8, 537 (1970).

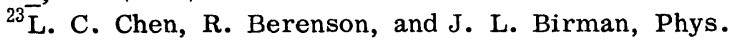
Rev. 170, 639 (1968).

${ }^{24}$ R. R. Hayes and K. H. Rieder, Phys. Rev. B $\underline{8}, 5972$ (1973). 